\title{
THE LEBESGUE DECOMPOSITION FOR GROUP-VALUED SET FUNCTIONS
}

\author{
BY \\ TIM TRAYNOR( $\left.{ }^{1}\right)$
}

\begin{abstract}
A Lebesgue-type decomposition is obtained for finitely additive set functions on a ring with values in topological groups. Corresponding results for Fréchet-Nikodým topologies are included. This generalizes Darst's result for real-valued set functions and a result of Drewnowski.
\end{abstract}

Introduction. Let $R$ be a ring of sets, and let $\mu$ and $\nu$ be finitely additive functions on $R$ with values in commutative topological groups. In [7], we saw that if $\mu$ is $s$-bounded we can give an adequate Lebesgue-type decomposition theorem for the algebraic type of absolute continuity ( $\mu$ is $\nu$-continuous in this sense if $\mu$ vanishes on $\nu$-null sets). This sort of decomposition is of primary interest when $\mu$ is $\sigma$-additive, $R$ is a $\sigma$-ring (or $\delta$-ring), and the range of $\nu$ is metrizable. The reason is that, in this case, the algebraic type of absolute continuity actually implies a topological continuity [2], [9] (roughly speaking, $\mu$ is $\nu$-continuous in this sense iff $\mu(A)$ is small whenever $\nu(E)$ is small for all $E$ in $R$ contained in $A$ ). For finitely additive functions, it is this condition which is really of interest. In the real-valued situation, Darst [1] proved that for bounded, real-valued additive functions, $\mu, \nu$, there is a Lebesgue decomposition for this kind of absolute continuity; namely, that $\mu$ can be uniquely written as the sum of bounded additive functions $\mu_{1}, \mu_{2}$ such that $\mu_{1}$ is $\nu$-continuous and $\mu_{2}$ is $\nu$-singular in the sense that for each $\epsilon>0$ there exists $E$ in $R$ such that for all $A$ in $R, \nu(A \cap E)<\epsilon$ and $\mu(A \backslash E)$ $<\epsilon$ (Darst assumed $R$ was an algebra, but this is no less general).

In [7], we obtained a Lebesgue-type decomposition for group-valued set functions using the topological type of $\nu$-continuity. Unfortunately, the singularity condition (called continuity nowhere) was formally weaker (cf. §3). Drewnowski [2] obtained a very nice decomposition for group-valued functions using a stronger notion of singularity, in the case the dominating set function has (pseudo) metrizable range. Drewnowski reminds us that (topological) $\nu$ continuity is the same as continuity in the uniform topology [2], [6] induced

Received by the editors October 2, 1974.

AMS (MOS) subject classifications (1970). Primary 28A45; Secondary 46G10.

Key words and phrases. Lebesgue decomposition, Hewitt-Yosida decomposition, vector measures, group-valued measures, finitely additive set functions, Fréchet-Nikodým topologies.

$\left({ }^{1}\right)$ This work was supported by the Summer Research Institute of the Canadian Mathematical Congress and by an N.R.C. Operating Grant. 
on $R$ by $\nu$ - if $\nu$ is nonnegative real valued, this is the topology of the metric $(A, B) \rightarrow v(A \Delta B)$ - and he takes this point of view in his decomposition. He thus decomposes $\mu$ with respect to a so-called Fréchet-Nikodým topology on $R$. (He still must assume that this topology is pseudometrizable, or is generated by an "additivity" $[3,2.1]$.)

In this article, we complete this circle of ideas, giving the analogue of Darst's theorem for group-valued additive set functions, and a corresponding decomposition for Fréchet-Nikodým topologies.

0 . Notation. Throughout the paper, $\mathbf{N}$ is the set of nonnegative integers; $Y$ and $Z$ are commutative topological groups (written additively); $V$ and $W$ are bases for the neighborhoods of 0 in $Y$ and $Z$, respectively, consisting of closed symmetric sets; $R$ is a ring of subsets of a space $S$.

For subsets $A, B$ of $S$, we write

$$
A B=A \cap B, \quad A \backslash B=\{a \in A: a \notin B\}, \quad A^{c}=S \backslash A, \quad A \Delta B=(A \backslash B) \cup(B \backslash A) .
$$

For any set function $\varphi$ on $R$,

$$
\varphi((A))=\{\varphi(E): A \supset E \in R\} .
$$

We assume that $\mu$ is finitely additive on $R$ to $Y$, and $\nu$ is finitely additive on $R$ to $Z$.

Note. Since we are dealing with additive functions, requiring that $Y$ and $Z$ are commutative is really no restriction: for $A, B$ in $R, \mu(A)+\mu(B)=\mu(A \cup B)+\mu(A B)=\mu(B)$ $+\mu(A)$. So $\mu$ has commutative range.

Usually we will impose the condition that $\mu$ be s-bounded [5], [7], that is, that $\mu\left(A_{i}\right) \rightarrow 0$ whenever $A=\left(A_{i}\right)_{i}$ is a disjoint sequence in $R$. Drewnowski [2], [3] used the term "exhaustive" for this concept.

Finally, for the purpose of $\epsilon / 2^{n}$-type arguments given $V$ in $V$, we choose, by continuity of addition, neighborhoods $V_{n}$ in $V$ such that for all $n, \Sigma_{i=0}^{n} V_{i}$ $\subset V$.

1. The main results. In this section we suppose that the topological group $Y$ is Hausdorff. We are interested here in the " $\epsilon, \delta$ "-type of absolute continuity and singularity:

1.1. Definitions. (1) $\mu$ is $\nu$-continuous iff for each $V$ in $V$, there exists $W$ in $W$ with $\mu(A) \in V$ whenever $A \in R$ and $\nu((A)) \subset W$.

(2) $\mu$ is $\nu$-singular iff for each $V$ in $V$ and $W$ in $W$, there exists $A$ in $R$ with $\nu((A)) \subset W, \mu\left(\left(A^{c}\right)\right) \subset V$.

Here is the Lebesgue decomposition for these notions:

1.2. THEOREM. Let $\mu$ be s-bounded and finitely additive on the ring $R$ to a complete subset of $Y$, and let $\nu$ be finitely additive on $R$ to $Z$. Then there exist unique s-bounded finitely additive functions $\mu_{1}, \mu_{2}$ on $R$ to $Y$ such that $\mu=$ 
$\mu_{1}+\mu_{2}, \mu_{1}$ is $\nu$-continuous, and $\mu_{2}$ is $\nu$-singular.

Both $\mu_{1}$ and $\mu_{2}$ are $\mu$-continuous.

In fact, we shall see that there are formulae for calculating the continuous and singular parts (2.2). Although our main interest is the above result in this form, we are going to reword it a bit so that we may answer a question of Drewnowski [3].

To each group-valued set function on $R$ corresponds a natural uniform topology on $R$.

1.3. Definitions. (1) For the set function $\nu$ on $R$ to $Z$, the $\nu$-topology on $R$ (denoted by $G_{\nu}$ ) is that topology on $R$ which has a base for the neighborhood system of a member $A$ of $R$ consisting of the families $\{B \in R: \nu((A \Delta B))$ $\subset W\}$, for $W$ in $W$.

(2) A topology $G$ on $R$ is called a Fréchet-Nikodým topology, provided that under this topology the map $(A, B) \rightarrow A \Delta B$ on $R \times R$ to $R$ is continuous and the map $A \rightarrow A B$ on $R$ to $R$ is continuous uniformly for $B$ in $R$.

The notion of Fréchet-Nikodým topology ("FN" topology) was introduced by Drewnowski [2] and studied in detail. (See also Sion [6].) Since we have assumed that $\nu$ is additive, it is evident that the $\nu$-topology on $R$ is an FN topology. Any FN topology on $R$ makes $R$ into a topological group and renders the map $(A, B) \rightarrow A B$ uniformly continuous. We should also point out that in an FN topology, $\varnothing$ has a base of neighborhoods $G$.with the added property that for each $A \in G, A E \in G$ for all $E$ in $R$.

Let $G$ be an FN topology on $R$. We see that $\mu$ is $G$-continuous iff for each $V$ in $V$ there is a neighborhood $G$ of $\varnothing$ in $G$ with $\mu(A) \in V$ for all $A$ in $G$.

An appropriate definition for $G$-singularity is :

1.4. Definition. $\mu$ is $G$-singular iff for each $V$ in $V$ and each $G$-neighborhood $G$ of $\varnothing$, there exists $A$ in $G$ with $\mu\left(\left(A^{c}\right)\right) \subset V$.

1.5. REMARKs. (1) Evidently, $\mu$ is $\nu$-continuous iff $\mu$ is $G_{\nu}$-continuous and $\mu$ is $\nu$-singular iff $\mu$ is $G_{\nu}$-singular.

(2) However, this "generalization" is only conceptual. Indeed, if $G$ is an FN topology on $R$, then $(R, \Delta)$ is a topological group under $G$ and, putting $\nu(A)$ $=A$ defines an additive map on $R$ to this group with $G_{\nu}=G$.

Now we can restate 1.2 so as to answer Drewnowski's question [3, p.47].

1.2'. THEOREM. Let $\mu$ be s-bounded and finitely additive on $R$ to a complete subset of $Y$ and $G$ an FN topology on $R$. Then, there exist unique sbounded finitely additive functions $\mu_{1}$ and $\mu_{2}$ on $R$ to $Y$ such that $\mu_{1}$ is G-continuous and $\mu_{2}$ is G-singular.

Both $\mu_{1}$ and $\mu_{2}$ are $\mu$-continuous. 
Actually, Drewnowski's definition of singularity is different from ours, but we will see that in the context of the theorem they are the same (Theorem 3.3).

2. The construction. Although it could be avoided, it is convenient to generate an FN topology by submeasures.

2.1. Definition (DRewnowski [2]). $q$ is a submeasure on $R$ iff $q$ is a function on $R$ to $[0, \infty)$ with $q(\varnothing)=0$ such that for $A, B$ in $R, q(A \cup B) \leqslant$ $q(A)+q(B)$ and $A \subset B$ implies $q(A) \leqslant q(B)$.

Drewnowski showed $[2, I, 2.7]$ that for any FN topology $G$ on $R$ there is a family $Q$ of continuous submeasures on $R$ which form a base for $G$ in the sense that the families $\{A \in R: q(A) \leqslant \epsilon\} \quad(q \in Q, \epsilon>0)$, form a base for the $G$ neighborhoods of $\varnothing$. The proof is easy: since $G$ makes $(R, \Delta)$ a topological group, there is a family $P$ of continuous invariant pseudometrics which generate $G$. For each $p$ in $P$, put

$$
\bar{p}(A)=\sup _{E \in R} p(A E, \varnothing)
$$

Take

$$
Q=\left\{\max _{p \in P^{\prime}} \bar{p}: P^{\prime} \text { is a finite subset of } P\right\} .
$$

Note that we can assume that $Q$ is directed to the right by $\leqslant: q_{1} \leqslant q_{2}$ iff $q_{1}(A) \leqslant q_{2}(A)$ for all $A$ in R. $\left({ }^{2}\right)$

From now on, $G$ is a fixed FN topology on $R ; Q$ is a base of submeasures for $G$, directed to the right by $\leqslant$; and for $q$ in $Q$, we put

$$
K_{q}=\left\{\alpha: \alpha=\left(\alpha_{n}\right)_{n} \text { is a decreasing sequence in } R \text { with } q\left(\alpha_{n}\right) \rightarrow 0\right\} \text {. }
$$

We note that $K_{q}$ is directed to the right by $<: \alpha<\beta$ iff $\alpha_{n} \subset \beta_{n}$ for all $n$ in $\mathbf{N}$.

The operators which give the decomposition $1.2^{\prime}$ can now be defined.

2.2. Definition. For $A$ in $R$ and $q$ in $Q$, put

$$
\begin{aligned}
c_{q} \mu(A) & =\lim _{\alpha \in K_{q}} \lim _{n} \mu\left(A \backslash \alpha_{n}\right), & s_{q} \mu(A) & =\lim _{\alpha \in K_{q}} \lim \mu\left(A \alpha_{n}\right) ; \\
c \mu(A) & =\lim _{q \in Q} c_{q} \mu(A), & s \mu(A) & =\lim _{q \in Q} s_{q} \mu(A),
\end{aligned}
$$

where $Q$ and $K_{q}$ are directed as indicated above-provided the limits exist.

$\left(^{2}\right)$ In case $Y$ is a topological vector space with topology generated by a family $S$ of seminorms and $\nu$ is finitely additive on $R$ to $Y$, the natural base of submeasures for the $\nu$ topology is obtained by assigning to each $s$ in $S$ the s-semivariation of $\nu, s \cdot \nu(A)=$ $\sup _{E \in R} S(\nu(A E))$ and putting $\left\{q \mid q=\max _{S \in S^{\prime}} s \cdot \nu, S^{\prime}\right.$ is a finite subset of $\left.S\right\}$. In case $Y$ is a group, quasinorms [2] are used instead of seminorms. 
Evidently, if one of $c_{q} \mu$ and $s_{q} \mu$ exists, so does the other and $\mu=c_{q} \mu+$ $s_{q} \mu$. Similarly for $c \mu$ and $s \mu$. Theorem 1.2' and a little more follows from the following lemmas (sba( $R, Y$ ) denotes the group (vector space if $Y$ is a topological vector space) of $s$-bounded finitely additive functions on $R$ to $Y$.)

2.3. Lemmas. Assume $Y$ is Hausdorff and complete and let $\mu \in \operatorname{sba}(R, Y)$.

(1) The limits defining $c_{q} \mu(A)$ and $s_{q} \mu(A)$ exist in $Y$, uniformly for $A$ in $R$.

(2) The limits defining $c \mu(A)$ and $\operatorname{s\mu }(A)$ exist in $Y$, uniformly for $A$ in $R$.

(3) $c \mu$ and su are $\mu$-continuous.

(4) $c$ and $s$ are additive (linear) operators on $\operatorname{sba}(R, Y)$ to $\operatorname{sba}(R, Y)$.

(5) If $\mu$ is G-continuous then $c \mu=\mu$, s $\mu=0$.

(6) If $\mu$ is G-singular then $s \mu=\mu, c \mu=0$.

(7) $c \mu$ is G-continuous.

(8) $s \mu$ is G-singular.

(9) If $\mu=\mu_{1}+\mu_{2}$ where $\mu_{1}, \mu_{2} \in \operatorname{sba}(R, Y), \mu_{1}$ is Gcontinuous and $\mu_{2}$ is Gsingular, then $\mu_{1}=c \mu, \mu_{2}=s \mu$.

In the case $Y$ is complete, Theorem $1.2^{\prime}$ follows immediately from 2.3 , the desired decomposition being $\mu=c \mu+s \mu$. If we only assume $\mu$ has its range in a complete subset of $Y$, we are not much worse off: Embed $Y$ in its completion $\bar{Y}$; then $c \mu(A)$ and $s \mu(A)$ exist in $\bar{Y}$ and have all the desired properties. But since the range of $\mu$ has complete closure, we see from their definitions that $c \mu$ and $s \mu$ actually take their values in $Y$.

Proofs of the Lemmas 2.3. By an easy indirect argument, which by now is quite -standard, one can see that $\mu$ is $s$-bounded iff for each disjoint sequence $E$ in $R, \mu\left(A E_{n}\right) \rightarrow 0$ uniformly for $A$ in $R$, or equivalently, since $Y$ is complete, iff for each increasing (or decreasing) sequence $\alpha$ in $R, \lim _{n} \mu\left(A \alpha_{n}\right)$ exists uniformly for $A$ in $R$. For these proofs, let us denote such a limit by $\bar{\mu}(A, \alpha)$.

(1) It is sufficient to show that, for each $q$ in $Q, s_{q} \mu(A)=\lim _{\alpha \in K_{q}} \bar{\mu}(A, \alpha)$ exists in $Y$, uniformly for $A$ in $R$. Fix $q$ in $Q$ and assume that the net $(\vec{\mu}(A, \alpha)$ : $\alpha \in K_{q}$ ) does not converge uniformly for $A$ in $R$. Then, for some $V$ in $V$, we can choose, for each $n$ in $\mathrm{N}, \alpha^{n}$ in $K_{q}$ with $\alpha^{n}<\alpha^{n+1}$ and

$$
\bar{\mu}\left(A, \alpha^{n}\right)-\bar{\mu}\left(A, \alpha^{n+1}\right) \notin V+V+V, \text { for some } A \text { in } R .
$$

Using s-boundedness (see the remark at the beginning of the proof) we choose recursively $k_{n}$ in $\mathbf{N}$, for $n$ in $\mathbf{N}$, with $k_{n}<k_{n+1}$ and $\mu\left(\left(\alpha_{k_{n}}^{n} \backslash \alpha_{k}^{n}\right)\right) \subset V_{n}$ for $k$ $\geqslant k_{n}$. This implies two things. First, since $V_{n}$ is closed, that

$$
\mu\left(A \alpha_{k_{n}}^{n}\right)-\bar{\mu}\left(A, \alpha^{n}\right) \in V_{n} \text { for all } A \text { in } R ;
$$

secondly, that for all $i \leqslant n, \mu\left(\left(\alpha_{k_{i}}^{i} \backslash \alpha_{k_{n}}^{n}\right)\right) \subset V_{i}$. [Indeed, for $i \leqslant n, \alpha^{i}<\alpha^{n}$ (so that $\left.\alpha_{k_{n}}^{i} \subset \alpha_{k_{n}}^{n}\right)$ and $k_{n} \geqslant k_{i}$, so $\mu\left(\left(\alpha_{k_{i}}^{i} \backslash \alpha_{k_{n}}^{n}\right)\right) \subset \mu\left(\left(\alpha_{k_{i}}^{i} \backslash \alpha_{k_{n}}^{i}\right)\right) \subset V_{i}$.] Now, put 
$B_{n}=\bigcup_{i=0}^{n} \alpha_{k_{i}}^{i}$. Then, since $\mu$ is additive,

$$
\mu\left(\left(B_{n} \backslash \alpha_{k_{n}}^{n}\right)\right)=\mu\left(\left(\bigcup_{i=0}^{n-1} \alpha_{k_{i}}^{i} \backslash \alpha_{k_{n}}^{n}\right)\right) \subset \sum_{i \leqslant n} \mu\left(\left(\alpha_{k_{i}}^{i} \backslash \alpha_{k_{n}}^{n}\right)\right) \subset V_{0}+\cdots+V_{n-1},
$$

which together with $(* *)$ yields

$$
\mu\left(A B_{n}\right)-\bar{\mu}\left(A, \alpha^{n}\right) \in V_{0}+\cdots+V_{n} \subset V,
$$

for all $A$ in $R$ and $n$ in N. But $\mu$ is s-bounded, so for large $n, \mu\left(A B_{n+1}\right)-$ $\mu\left(A B_{n}\right) \in V$ for all $A$ in $R$. Thus,

$$
\begin{aligned}
\bar{\mu}\left(A, \alpha^{n}\right)-\bar{\mu}\left(A, \alpha^{n+1}\right)= & \bar{\mu}\left(A, \alpha^{n}\right)-\mu\left(A B_{n}\right)+\mu\left(A B_{n}\right)-\mu\left(A B_{n+1}\right) \\
& +\mu\left(A B_{n+1}\right)-\bar{\mu}\left(A, \alpha^{n+1}\right) \\
\in & V+V+V,
\end{aligned}
$$

for all $A$ in $R$. This contradicts (*) and proves (1).

(2) It is sufficient to show that the net $\left(s_{q} \mu(A): q \in Q\right)$ converges uniformly for $A$ in $R$. The proof is similar to that of (1) but a little more delicate:

If the conclusion were false, then for some $V$ in $V$ we could choose, for each $n$ in $\mathbf{N}, q_{n}$ in $Q$ such that $q_{n} \leqslant q_{n+1}$ and:

$$
s_{q_{n}} \mu(A)-s_{q_{n+1}} \mu(A) \notin V+V+V, \text { for some } A \text { in } R \text {. }
$$

For each $n$, find $\alpha^{n}$ in $K_{q_{n}}$ such that for all $\alpha$ in $K_{q_{n}}$ with $\alpha>\alpha^{n}, \bar{\mu}(A, \alpha)-$ $\bar{\mu}\left(A, \alpha^{n}\right) \in V_{n}$ for all $A$ in $R$. Since $V_{n}$ is closed, we also have

$$
s_{q_{n}} \mu(A)-\bar{\mu}\left(A, \alpha^{n}\right) \in V_{n} \quad \text { for } A \text { in } R .
$$

Now, if $\alpha$ is any member of $K_{q_{n}}$, then the sequence $\alpha \cup \alpha^{n}=\left(\alpha_{k} \cup \alpha_{k}^{n}\right)_{k}$ also belongs to $K_{q_{n}}$ and $\alpha \cup \alpha^{n}>\alpha^{n}$, so we have

$$
\lim _{k} \mu\left(A \alpha_{k} \mid \alpha_{k}^{n}\right)=\bar{\mu}\left(A, \alpha \cup \alpha^{n}\right)-\bar{\mu}\left(A, \alpha^{n}\right) \in V_{n} .
$$

If $i \leqslant n$, then $q_{i} \leqslant q_{n}$, so $\alpha^{n} \in K_{q_{n}} \subset K_{q_{i}}$, and by the last sentence, $\lim _{k} \mu\left(A \alpha_{k}^{n} \backslash \alpha_{k}^{i}\right) \in V_{i}$ for all $A$ in $R$. Putting $\beta_{k}^{n}=\bigcap_{i<n} \alpha_{k}^{i}$, we have for all $A$ in $R$,

$$
\bar{\mu}\left(A, \alpha^{n}\right)-\bar{\mu}\left(A, \beta^{n}\right)=\lim _{k} \mu\left(\bigcup_{i=0}^{n-1}\left(\alpha_{k}^{n} \backslash \alpha_{k}^{i}\right)\right) \in V_{0}+\cdots+V_{n-1},
$$

so that using (**), $s_{q n} \mu(A)-\bar{\mu}\left(A, \beta^{n}\right) \in V_{0}+\cdots+V_{n}$. Now, choose for each $n, k_{n}$ in $\mathrm{N}$ with $k_{n} \leqslant k_{n+1}$ and $\bar{\mu}\left(A, \beta^{n}\right)-\mu\left(A \beta_{k_{n}}^{n}\right) \in V_{n+1}$, for all $A$ in $R$. Then

$(* * *)$

$$
s_{q_{n}} \mu(A)-\mu\left(A \beta_{k_{n}}^{n}\right) \in V_{0}+\cdots+V_{n}+V_{n+1} \subset V .
$$

Moreover, for each $n, \beta_{k_{n}}^{n} \supset \beta_{k_{n+1}}^{n+1}$ and $\mu$ is s-bounded, so that for large $n$, 
$\mu\left(A \beta_{k_{n}}^{n}\right)-\mu\left(A \beta_{k_{n+1}^{n+1}}^{n+1}\right) \in V$ for all $A$ in $R$. Combined with (***) this contradicts $(*)$ and (2) is proved.

(3) For each $A$ in $R, V$ in $V$, if $\mu((A)) \subset V$ then $c \mu(A)=$ $\lim _{q} \lim _{\alpha} \lim _{n} \mu\left(A \backslash \alpha_{n}\right) \in V$, since $V$ is closed. Thus $c \mu$ is $\mu$-continuous. Similarly for $s \mu$.

(4) By continuity of addition in $Y$, we see that $c \mu$ and $s \mu$ are finitely additive. Since each is $\mu$-continuous, each is clearly $s$-bounded also. Thus $c$ and $s$ map $\operatorname{sba}(R, Y)$ into itself. The additivity (linearity) of $c$ and $s$ also follow from continuity of the operation(s) in $Y$.

(5) Suppose $\mu$ is G-continuous. Given $V$ in $V$, find $q_{0}$ in $Q$ and $\epsilon>0$ such that $q_{0}(A) \leqslant \epsilon$ implies $\mu(A) \in V$. If $q \geqslant q_{0}$, for each $\alpha$ in $K_{q}$ we have for large $n, q_{0}\left(A \alpha_{n}\right) \leqslant q\left(\alpha_{n}\right) \leqslant \epsilon$ and hence $\mu\left(A \alpha_{n}\right) \in V$, for all $A$ in R. Letting $n$ and then $\alpha$ run, we have $s_{q} \mu(A)$ in $V$ for $q \geqslant q_{0}, A \in R$. This proves $s \mu=0$ and hence $c \mu=\mu$.

(6) Suppose $\mu$ is G-singular and let $V \in V, q \in Q$. For each $n$ in $\mathbf{N}$, choose $A_{n}$ in $R$ with $q\left(A_{n}\right)<1 / n+1$ and $\mu\left(\left(A^{c}\right)\right) \subset V_{n}$. Put $\alpha_{n}=\bigcap_{i=0}^{n} A_{i}$. Then $\alpha \in K_{q}$, and we can easily check that for $\beta>\alpha, \lim _{n} \mu\left(A \backslash \beta_{n}\right) \in V$, for all $A$ in $R$. Thus $c_{q} \mu(A) \in V$ for arbitrary $V$ in $V$ and $q$ in $Q$, whence $c \mu=0$ and $s \mu=\mu$.

(7) To show that $c \mu$ is continuous, first let $q \in Q$ and let $A$ be a decreasing sequence in $R$ with $q\left(A_{i}\right)>0$; that is, let $A \in K_{q}$. Since the limits defining $c_{q} \mu\left(A_{i}\right)$ are uniform in $i$, we have $\lim _{i} c_{q} \mu\left(A_{i}\right)=\lim _{\alpha} \lim _{n} \lim _{i} \mu\left(A_{i} \backslash \alpha_{n}\right)$. But for $\alpha>A, A_{i} \backslash \alpha_{n}=\varnothing$ for $i \geqslant n$, so this limit is 0. By Drewnowski [2, II, Theorem 6.1 ], this shows that $c_{q} \mu$ is $q$-continuous; that is, continuous in the topology induced on $R$ by $q$. Since $q$ is itself G-continuous, $c_{q} \mu$ is G-continuous. But according to (2), $\lim _{q} c_{q} \mu(A)$ exists uniformly in $A$. Hence, if $\left(A_{i}\right)_{i}$ is a net converging to $\varnothing$ in $G$, we have $\lim _{i} c \mu\left(A_{i}\right)=\lim _{q} \lim _{i} c_{q} \mu\left(A_{i}\right)=0$, as claimed.

(8) Since $c \mu$ is continuous and $s$-bounded we can apply (5) to get $s(c \mu)=$ 0 . But then $s \mu=s c \mu+s s \mu=s s \mu$. Thus, it is sufficient to show that $s \mu=\mu$ implies $\mu$ is G-singular. Accordingly, suppose $s \mu=\mu$. We notice first that if $V \in$ $V, B \in R$ and $\mu(B) \notin V$, then for each $q_{0}$ in $Q$ and $\epsilon>0$ there exists $B^{\prime}$ in $R$ contained in $B$ such that $\mu\left(B^{\prime}\right) \notin V$ and $q_{0}\left(B^{\prime}\right) \leqslant \epsilon(*)$. [Indeed, if $\lim _{q} \lim _{\alpha} \lim _{n} \mu\left(B \alpha_{n}\right)=s \mu(B)=\mu(B) \notin V$, a closed set, then there exists $q \geqslant$ $q_{0}$, and $\alpha$ in $K_{q}$, such that for large $n \mu\left(B \alpha_{n}\right) \notin V$. Choose $n$ large enough that $q\left(\alpha_{n}\right) \leqslant \epsilon$ and take $B^{\prime}=B \alpha_{n}$.]

Now, suppose $\mu$ is not singular. Then there exists $V$ in $V, q$ in $Q$ and $\epsilon>$ 0 such that, whenever $A \in R$ and $q(A) \leqslant \epsilon, A^{c}$ contains some $B$ in $R$ with $\mu(B)$ $\notin V$. Start with $A=\varnothing$ and combine this fact with (*) to find $A_{0}$ in $R$ with $\mu\left(A_{0}\right) \notin V, q\left(A_{0}\right) \leqslant \epsilon / 2$. Recursively, suppose $A_{0}, \ldots, A_{n}$ are disjoint members of $R$, chosen so that $\mu\left(A_{i}\right) \notin V, q\left(A_{i}\right) \leqslant \epsilon / 2^{i+1}$, for $i=0, \ldots, n$. Then $q\left(A_{0} \cup \cdots \cup A_{n}\right) \leqslant \epsilon$, so that $\left(A_{0} \cup \cdots \cup A_{n}\right)^{c}$ contains a member $A_{n+1}$ of 
$R$ with $\mu\left(A_{n+1}\right) \notin V$ which by (*) may be chosen so that $q\left(A_{n+1}\right) \leqslant \epsilon / 2^{n+2}$. The disjoint sequence thus constructed contradicts the hypothesis that $\mu$ is sbounded, so $\mu$ is singular.

(9) If $\mu=\mu_{1}+\mu_{2}$ where $\mu_{1}, \mu_{2}$ are as stated, we apply (5) and (6) to get $c \mu=c \mu_{1}+c \mu_{2}=\mu_{1}$ (and $s \mu=\mu_{2}$ ).

3. Singularity. In the next three sections, we consider some corollaries and extensions of the above results. We continue to assume that $\mu$ is a finitely additive function on the ring $R$ to the commutative topological group $Y$ and that $G$ is an FN topology on $R$. In addition, we introduce the following terminology and notation.

3.1. Definitions. For $A$ in $R$,

(1) $A$ is $\mu$-null iff $\mu(E) \in$ closure $\{0\}$, whenever $A \supset E \in R$;

(2) $\mu_{A}$ is the map $E \rightarrow \mu(A E)$ on $R$ to $Y$.

For FN topologies $T_{1}, T_{2}$ on $R$,

(3) $T_{1} \vee T_{2}$ is the smallest (FN) topology containing $T_{1}$ and $T_{2}$.

(4) $T_{1} \wedge T_{2}$ is the largest FN topology contained in $T_{1}$ and $T_{2}$.

(5) $O$ denotes $\{\varnothing, R\}$, the trivial topology on $R$.

In this section, we wish to compare our definition of singularity with two related notions.

3.2. Definitions. (1) $\mu$ is nowhere G-continuous iff each $A$ in $R$ for which $\mu_{A}$ is G-continuous is $\mu$-null.

(2) $\mu$ is extremely non-G-continuous iff $G_{\mu} \wedge G=0$.

In [7, Theorem 2.4] we used the first notion to obtain one version of the Lebesgue decomposition. (In that paper we talked only about continuity with respect to a group-valued additive set function, but in view of 1.4(2), this is no less general.) Drewnowski [3] used the second notion (which he referred to both as "extreme noncontinuity" and as "singularity") to obtain a Lebesgue decomposition in the case where $G$ was generated by one submeasure or by an "additivity". In general, singular $\Rightarrow$ extremely noncontinuous $\Rightarrow$ nowhere continuous, but in the context of 1.2 or $1.2^{\prime}$-where $\mu$ is assumed s-bounded-we find that all three notions coincide! Unfortunately, it appears that we need our present construction to prove this, otherwise we could just apply the above mentioned result from [7] to obtain the decompositions of $\$ 1$. The definition used in this paper was chosen because it seems to give more insight into the nature of singularity, it is probably more useful, and it more closely resembles the definition often used in the real-valued case [1].

3.3. THEOREM. Let $\mu: R \rightarrow Y$ be finitely additive, and let $G$ be an $F N$ topology on $R$.

(1) If $\mu$ is G-singular, then $\mu$ is extremely non-G-continuous. 
(2) If $\mu$ is extremely non-Gcontinuous, then $\mu$ is nowhere G-continuous.

(3) In case $\mu$ is s-bounded, all three conditions coincide.

Before proving this, we note that the concepts involved only depend on the $\mu$-topology $G_{\mu}$, not on $\mu$ itself:

3.4. Lemmas. (1) If $\mu_{1}$ and $\mu_{2}$ are topological group-valued additive set functions which are equivalent in the sense that $G_{\mu_{1}}=G_{\mu_{2}}$, then $\mu_{1}$ is Gcontinuous (or G-singular, or extremely non-G-continuous, or nowhere G-continuous, or s-bounded) iff $\mu_{2}$ is.

(2) Given any topological group-valued additive set function on $R$, there is an equivalent one with range in a complete Hausdorff group.

PROoF. (1) This is straightforward and is left to the reader. However, for future use, we note that $\mu$ is G-continuous if and only if $G_{\mu} \subset G$.

(2) Suppose $\mu$ is additive on $R$ to $Y$. Let $Y_{0}$ be the Hausdorff group obtained by identifying $x$ and $y$ iff $x-y \in$ closure $\{0\}$, let $\pi$ be the canonical homomorphism of $Y$ onto $Y_{0}$, and put $\bar{\mu}=\pi \circ \mu$. If $V$ is an open neighborhood of 0 in $Y$ then $\pi[V]$ is a neighborhood of the origin in $Y_{0}$ with $\pi^{-1}[\pi[V]=$ $V$; conversely, if $U$ is a neighborhood of the origin in $Y_{0}, \pi^{-1}[U]$ is a neighborhood of 0 in $Y$, with $\pi\left[\pi^{-1}[U]=U\right.$. From this, we can easily check that $G_{\mu}$ $=G_{\bar{\mu}}$. Of course, completing $Y_{0}$ will not change the topology $G_{\bar{\mu}}$, so we are done.

Proof OF 3.3. (1) Let $\mu$ be $G$-singular and suppose that $G$ is a neighborhood of $\varnothing$ in $G_{\mu} \wedge G$. By definition of FN topology (1.3(2)), there is another neighborhood $G_{0}$ of $\varnothing$ in $G_{\mu} \wedge G$ with $G_{0} \dot{\Delta} G_{0}=\left\{A \Delta B: A, B \in G_{0}\right\} \subset G$ and $\left\{A B: A \in G_{0}, B \in R\right\} \subset G_{0}$. Since $G_{0}$ is a $G_{\mu}$-neighborhood of $\varnothing$, there exists $V$ in $V$ such that $\{E \in R: \mu((E)) \subset V\} \subset G_{0}$. Fix such a $V$. Since $G_{0}$ is a $G$-neighborhood of $\varnothing$ and $\mu$ is $G$-singular, there exists $A$ in $G_{0}$ with $\mu\left(\left(A^{c}\right)\right) \subset$ $V$. Thus, for all $B$ in $R, B=B A \cup B A^{c} \in G_{0} \dot{\Delta} G_{0} \subset G$. This shows that $R$ is the only neighborhood of $\varnothing$ in $G_{\mu} \wedge G$, so $\mu$ is extremely non-G-continuous.

(2) Suppose $\mu$ is extremely non-G-continuous. If $A \in R$ and $\mu_{A}$ is Gcontinuous, then $G_{\mu_{A}} \subset G_{\mu} \wedge G$, so $G_{\mu_{A}}=0=\{\varnothing, R\}$. This means that for all $B$ in $R$ and all $V$ in $V, \mu(A B)=\mu_{A}(B) \in V$. Thus $\mu((A)) \in$ closure $\{0\}$ and $A$ is $\mu$-null.

(3) By Lemma 3.4, we may assume $Y$ is Hausdorff and complete. If $\mu$ is s-bounded and nowhere $\mu$-continuous, write $\mu=c \mu+s \mu$, where $c \mu$ is G-continuous and $s \mu$ is $G$-singular. Since $s \mu$ is also nowhere continuous by (1) and (2), we see that $c \mu$ is both continuous and nowhere continuous. Thus each $A$ in $R$ is $c \mu$-null, so $\mu(A)=c \mu(A)+s \mu(A)=s \mu(A)$; whence, $\mu$ is singular. 


\section{Decomposition of FN topologies.}

4.1. Definition. For an FN topology $T$ on $R, T$ is called exhaustive or s-bounded iff each disjoint sequence in $R$ converges in $T$ to $\varnothing$.

Along with his question on decomposition of additive set functions, Drewnowski [3, p. 47] asks whether exhaustive FN topologies can be similarly decomposed. We see now that the answer is "yes".

4.2. THEOREM. Let $T$ be an exhaustive $F N$ topology on $R$ and $G$ any other FN topology on $R$. Then there exist unique $F N$ topologies $T_{1}$ and $T_{2}$ on $R$ with $T=T_{1} \vee T_{2}, T_{1} \subset G$, and $T_{2} \wedge G=0$.

Proof. Think of $(R, \Delta)$ as a topological group $(Y,+)$ under the topology $T$. The identity map $\mu: R \rightarrow Y(\mu(A)=A)$ is clearly finitely additive and sbounded, with $G_{\mu}=T$. By Lemma 3.4, there exists an equivalent additive $\bar{\mu}$ on $R$ to some complete Hausdorff topological group $\bar{Y}$. Write $\bar{\mu}=c \bar{\mu}+s \bar{\mu}$, the decomposition into $G$-continuous and $G$-singular parts. Put $T_{1}=G_{c \bar{\mu}}$ and $T_{2}=$ $G_{s \bar{\mu}}$. Then $T_{1} \subset G$ and, by 3.3(1) $T_{2} \wedge G=0$. Since both $c \bar{\mu}$ and $s \bar{\mu}$ are $\bar{\mu}$ continuous, $T_{1} \vee T_{2} \subset T$. On the other hand, since $\bar{\mu}=c \bar{\mu}_{1}+s \bar{\mu}_{2}$ we easily check that $T=G_{\bar{\mu}} \subset T_{1} \vee T_{2}$.

5. The Hewitt-Yosida decomposition; additivities. We now consider the problem of representing an additive function as the sum of a $\sigma$-additive and a "purely finitely additive" one. The notation of $\$ 0$ is still assumed.

5.1. Definition. For a finitely additive function $\mu$ on $R$ to $Y, \mu$ is purely finitely additive iff $\mu$ is $\nu$-singular for each $\sigma$-additive function $\nu$ on $R$ to a (commutative) topological group.

With this definition, we can state the following Hewitt-Yosida-type theorem.

5.2. Theorem. Assume $Y$ is Hausdorff and let $\mu$ be finitely additive on $R$ to a complete subset of $Y$. Then there exist unique s-bounded finitely additive functions $\mu_{1}$ and $\mu_{2}$ such that $\mu=\mu_{1}+\mu_{2}, \mu_{1}$ is $\sigma$-additive and $\mu_{2}$ is purely finitely additive.

In [8], we obtained such a theorem, with a slightly weaker definition of "purely finitely additive"; namely, " $\mu$ is $\nu$-singular for each $s$-bounded finitely additive function $\nu$ on $R$ to a commutative topological group". The s-boundedness of $\nu$ was superfluous to the proof. Its use $[8$, p. 1168, 1. 19] can be replaced by appealing to Lemma 5.3 below. We now feel that the present definition is the "right one". (Of course, by arguments such as those in $\S 3$, we see that for $\mu$ s-bounded the two conditions coincide.)

5.3. LEMMA. Let $\nu$ be finitely additive on $R$ to $Z$. Then $\nu$ is $\sigma$-additive iff for each decreasing sequence $A$ in $R$ with $\bigcap_{n} A_{n}=\varnothing, \nu\left(E A_{n}\right) \rightarrow 0$, uniformly for $E$ in R; i.e. $A_{n} \rightarrow \varnothing$ in $G_{\nu}$. 
Proof. The condition is clearly sufficient. On the other hand, if the condition were not satisfied, then for some $W$ in $W$, there would exist a subsequence $B$ of $A$ and a sequence $E$ in $R$ with $\nu\left(E_{n} B_{n}\right)-\nu\left(E_{n} B_{n+1}\right) \notin W$, for all n. Rewrite this: $\nu\left(E_{n} B_{n} \cup B_{n+1}\right)-\nu\left(B_{n+1}\right) \notin W$. But each of these terms tends to 0 by $\sigma$-additivity, a contradiction.

Theorem 5.2 fits into the context of $\S \S 1$ and 2 as follows. We could replace the $K_{q}$ 's of Definition 2.2 by one family $K$, the set of all decreasing sequences in $R$ with void intersection. Then the $\sigma$-additive and purely finitely additive parts are given by $\lim _{\alpha \in K} \lim _{n} \mu\left(A \backslash \alpha_{n}\right), \lim _{\alpha \in K} \lim _{n} \mu\left(A \alpha_{n}\right)$, respectively. (The third iterated limit being unnecessary.) This method is actually very close to that of [8] and of [3]. (See Remarks 5.4 below.) If it is preferred, we could regard 5.2 as a special case of $1.2^{\prime}$.

Call an FN topology $G$ on $R$ order continuous [2, III, p. 439] iff for each decreasing sequence $A$ in $R$ with $\bigcap_{n} A_{n}=\varnothing, A_{n} \rightarrow 0$ in $G$. There is a largest such topology. This follows from [2, III, 8.1], but it is easy to check directly: let $\tau$ be the set of all order continuous FN topologies and see that the topology $G_{0}$ generated by $\bigcup_{G \in \tau} G$ is also an order continuous FN topology, hence the largest one. Theorem 5.2 now follows from $1.2^{\prime}$ and the following lemmas.

LEMmas. (1) $\mu$ is $G_{0}$-continuous iff $\mu$ is $\sigma$-additive.

(2) $\mu$ is $G_{0}$-singular iff $\mu$ is purely finitely additive.

We omit the easy proofs.

5.4. Additivities. The proof of the Hewitt-Yosida decomposition given in [8] involved the generation of an outer measure and restricting it to $R$. If we confine our attention to $R$, the construction looks like this: To each $A$ in $R$, correspond the family $S[A]$ of countable partitions of $A$ by members of $R$, directed by refinement. The $\sigma$-additive part of $\mu$ is given by $\mu_{1}(A)=$ $\lim _{P \in G[A]} \Sigma_{E \in P} \mu(E)$, and the purely finitely additive part by $\mu_{2}(A)=\mu(A)-$ $\mu_{1}(A)$. Drewnowski [3] also noticed this (independently) and introduced the notion of an additivity, a generalization of the notion of the partitions just mentioned. This enabled him to neatly prove the Hewitt-Yosida decomposition and the Lebesgue decomposition simultaneously. The latter was obtained only for the case that $\nu$ takes its values in a pseudometrizable group. We now see that it could have been done in general, using families of additivities. Indeed, we could have replaced in 2.2 the family $K_{q}$ by the $q$-additivity $\mathfrak{S}_{q}[3$, p. 29] and obtained $c_{q} \mu(A)=\lim _{P \in G_{q}[A]} \Sigma_{E \in P} \mu(E)$. The $K_{q}$ are, however, technically easier to handle.

6. Remarks.

6.1. THE $\sigma$-ADDITIVE CASE: THE METHOD OF IDEALS. Here we wish to outline how the method of $\S 2$ simplifies if $\mu$ is $\sigma$-additive on a $\sigma$-ring $R$ (a $\delta$-ring would 
do). Assume $Y$ is Hausdorff and complete and that $Q$ is a base of submeasures for an FN topology on R. For each $q$ in $Q$, let $N_{q}=\{A \in R: q(A)=0\}$. Then $N_{q}$ is an ideal in $R$ and the G-continuous and G-singular parts of $\mu$ are given by

$$
c \mu(A)=\lim _{q \in Q} \lim _{E \in N_{q}} \mu(A \backslash E), \quad s \mu(A)=\lim _{q \in Q} \lim _{E \in N_{q}} \mu(A E),
$$

where $N_{q}$ is directed upward by inclusion and $Q$ is directed as in $\S 2$. (This is a natural extension of the method of [7].) In this framework, the relevant lemmas corresponding to 2.3 are somewhat easier to prove.

6.2. ThE ADDITIVE CASE - FROM THE $\sigma$-ADDITIVE ONE. Now, suppose one is interested in the decomposition theorem $1.2^{\prime}$ but not in formulae such as those in 2.2. We could proceed as follows. Given a finitely additive s-bounded function $\mu$ on a ring $R$ to a complete subset of the Hausdorff group $Y$. For simplicity, take $Y$ complete and $R$ to be a field (if necessary, put $\mu\left(A^{c}\right)=-\mu(A)$ for $A$ in $R$ to extend $\mu$ from a ring to a field). Now use a standard trick:

Let $\pi$ be the Boolean isomorphism of $R$ onto its Stone representation $\bar{R}$ [4, p. 41]. Define $\bar{\mu}$ on $\bar{R}$ by $\bar{\mu}(A)=\mu\left(\pi^{-1}(A)\right)$. The function $\bar{\mu}$ is automatically $\sigma$-additive-every decreasing sequence in $\bar{R}$ is eventually constant-so $\bar{\mu}$ may be extended to a $\sigma$-additive function on the $\sigma$-field $\overline{\bar{R}}$ generated by $\bar{R}[6],[8]$. (Here s-boundedness is used.) The isomorphism $\pi$ also transfers $G$ to an FN topology $\bar{G}$ on $\bar{R}$ and this can be extended [2, III, 8.3] to an FN topology $\overline{\bar{G}}$ on $\overline{\bar{R}}$. Now, use 6.1 to decompose $\overline{\bar{\mu}}$ into $\overline{\bar{G}}$-continuous and $\overline{\bar{G}}$-singular parts $c \overline{\bar{\mu}}$ and $s \overline{\bar{\mu}}$. Put $\mu_{1}(A)=c \overline{\bar{\mu}}(\pi(A))$ and $\mu_{2}(A)=s \overline{\bar{\mu}}(\pi(A))$ for $A$ in $R$. Then $\mu=$ $\mu_{1}+\mu_{2}$ is the desired decomposition.

Although the method of $6.1,6.2$ is much less elementary than that of $\S 2$, it is the one which first led to the discovery of Theorem 1.2, and thence to the present paper.

6.3. ThE local SETTING. There has lately been considerable interest in looking at what happens if a property of set functions holds only locally. A map $\mu: R \rightarrow Y$ has a property locally iff the map $\mu_{A}: R \rightarrow Y$ defined by $\mu_{A}(E)=$ $\mu(A E)$ has this property, for each $A$ in $R$. Much of the above work has a local analogue. For example, Theorem $1.2^{\prime}$ can be modified to read 'If $\mu$ is locally $s$-bounded finitely additive on $R$ to a complete subset of $Y$, then there exist unique locally s-bounded finitely additive functions $\mu_{1}, \mu_{2}$ such that $\mu=\mu_{1}+$ $\mu_{2}, \mu_{1}$ is locally G-continuous, and $\mu_{2}$ is locally G-singular". The usual trick works: Find $c \mu_{A}$ and $s \mu_{A}$ for $A$ in $R$ and put $\mu_{1}(A)=c \mu_{A}(A), \mu_{2}(A)=$ $s \mu_{A}(A)$.

It is perhaps worth pointing out that $\mu$ is locally $s$-bounded iff for each decreasing sequence $A$ in $R,\left(\mu\left(A_{n}\right)\right)_{n}$ is a Cauchy sequence in $Y$. 
ADDED IN PROOF. 1. The suggestive terminology "sideways continuous" is used by Alexiuk and Beznosikov instead of "s-bounded" or "exhaustive". This is probably better, since s-bounded (which is short for "stronglybounded") only implies bounded for additive set functions and only "exhausts" when the corresponding FN topology is metrizable.

2. Of interest in connection with this paper are:

T. P. Dence, $A$ Lebesgue decomposition for vector valued additive set functions, Pacific J. Math. 57 (1975), 91-98, and

R. B. Darst, The Lebesgue decomposition for lattices of projection operators, Advances in Math. 1 (1975), 30-33.

\section{REFERENCES}

1. R. B. Darst, $A$ decomposition of finitely additive set functions, J. Reine Angew. Math. 210 (1962), 31-37. MR 25 \#1257.

2. L. Drewnowski, Topological rings of sets, continuous set functions, integration. I, II, III, Bull. Acad. Polon. Sci. Sér. Sci. Math. Astronom. Phys. 20 (1972), 269-276, 277286, 439-445. MR 46 \#5558; 47 \#5200. 49 \#518.

3. - Decompositions of set functions, Studia Math. 48 (1973), 23-48. MR

4. N. Dunford and J. T. Schwartz, Linear operators. I: General theory, Pure and Appl. Math., vol. 7, Interscience, New York, 1958. MR 22 \#8302.

5. C. E. Rickart, Decomposition of additive set functions, Duke Math. J. 10 (1943), 653-665. MR 5, 232.

6. M. Sion, Outer measures with values in a topological group, Proc. London Math. Soc. (3) 19 (1969), 89-106. MR 39 \#398.

7. T. Traynor, Decomposition of group-valued additive set functions, Ann. Inst. Fourier (Grenoble) 22 (1972), fasc. 3, 131-140. MR 48 \#11439.

8. - A general Hewitt-Yosida decomposition, Canad. J. Math. 24 (1972), 1164-1169. MR 47 \#3636.

9. (1973), 577-579. MR 50 \#7475.

DEPARTMENT OF MATHEMATICS, UNIVERSITY OF WINDSOR, WINDSOR, ONTARIO, CANADA 